\title{
Levels, Distribution and Sources of Polycyclic Aromatic Hydrocarbons in Surface Water in the Lower Reach of Qua Iboe River Estuary, Nigeria
}

\author{
Eno Anietie Moses ${ }^{*}$, Bassey Anie Etuk, Essien Daniel Udosen \\ Department of Chemistry, University of Uyo, Uyo, Nigeria \\ Email address: \\ enomoses27@yahoo.com (E. A. Moses), basseyetuk2@gmail.com (B. A. Etuk), essienandong@yahoo.com (E. D. Udosen)
}

\section{To cite this article:}

Eno Anietie Moses, Bassey Anie Etuk, Essien Daniel Udosen. Levels, Distribution and Sources of Polycyclic Aromatic Hydrocarbons in Surface Water in Lower Reach of Qua Iboe River Estuary, Nigeria. American Journal of Environmental Protection.

Vol. 4, No. 6, 2015, pp. 334-343. doi: 10.11648/j.ajep.20150406.20

\begin{abstract}
The lower reach of Qua Iboe River estuary houses one of the largest crude oil production facility in the Niger Delta region of Nigeria. Frequent oil spills has led to severe deterioration of the water system, This study determined 16 polycyclic hydrocarbons (PAHs) in surface waters of sites across the estuary in the wet and dry seasons. Gas chromatography with flame ionization detector (GC / FID) was used to measure PAHs in water samples following extraction. The sum of PAHs ranged from7.827E-04 for the wet season to $1.500 \mathrm{E}-02 \mathrm{mg} / 1$ for the dry season with significantly higher $(\mathrm{P}<0.05) \mathrm{PAH}$ concentration in the wet season. Total PAHs for the water sample at all sampling sites were above the permissible limit. The composition pattern of PAHs were dominated by high molecular weight PAHs, constituting 4-6 ring compounds. Diagnostic ratios of Fluoranthene/Fluoranthene + Pyrene, Anthracene/Anthracene + Phenanthrene and low molecular weight-PAH to high molecular weight PAH were calculated to evaluate possible sources of PAH compounds These ratios reflected predominantly pyrogenic sources of PAH to the water body originating from gas flaring from a petrochemical facility located close to the sampled stations. Cluster analysis was used to assess similarities between individual PAHs in water from QIRE for the dry and wet seasons. It revealed three primary PAH clusters in both seasons indicating different levels of anthropogenic activities in the cluster areas. There is a need for appropriate regulatory legislation on the control, treatment and discharge of oilfield effluents into QIRE and its adjourning creeks.
\end{abstract}

Keywords: Polycyclic Aromatic Hydrocarbon, Water Pollution, Qua Iboe River Estuary, Gas Flaring, Oil Pollution

\section{Introduction}

Water pollution occurs when the quality of water is changed in a way which affects the organisms that depend on it, or its suitability for recreational and domestic purposes [1]. Pollution of water is enhanced by industrialization, urbanization and technological development as these processes result in increased discharge of pollutants to receiving water, causing undesirable effects to the aquatic environment. Some contaminants of water include trace metals, pesticides and polycyclic aromatic hydrocarbons. Polycyclic aromatic hydrocarbons (PAHs), are formed by incomplete combustion of organic matter containing fuels such as oil, wood, coal, diesel, fat, garbage and incense [2]. PAHs occur naturally in coal, crude oil, and gasoline and are present in products made from fossil fuels such as asphalt, creosote, coal tar. Natural sources of PAHs include oil seeps, volcanic eruption, forest fires, decaying organic matter while anthropogenic sources include emission from petroleum refineries, gas flaring, power generation from fossil fuels, atmospheric fall out, inadvertent oil spill [3, 4]. PAHs are classified as low molecular weight (LMW), which constitute the 2 to 3 ringed compounds, and high molecular weight (HMW) of 4 to 6 rings. HMW PAHs are of significant concern because of their carcinogenicity, mutagenic properties and bioaccumulation in organic tissues due to their lipophilic character. Prolonged exposure to low levels of PAHs leads to adverse health effects due to their toxicity, carcinogenicity and mutagenicity $[5,6]$. rude oil provides $20 \%$ of Nigeria's gross domestic product, $65 \%$ of its budgetary revenues and $95 \%$ of the country's export earnings [7]. However, the exploration, refining and 
exploitation of crude oil have resulted in environmental degradation which affects communities near and far away from offshore and onshore installations of oil companies. Usually oil exploration activities have resulted in the release of large amounts of hydrocarbons into the terrestrial and aquatic environment (8). This has led to disruption of socio-economic activities, water scarcity, poor esthetics and deterioration of water quality in rivers of the Niger Delta [9]. Qua Iboe terminal (QIT) located at the Qua Iboe River estuary, is one of the largest crude oil production facility in the Niger Delta region of Nigeria [10]. Activities at the terminal has facilitated the establishments of smaller oil servicing companies at the estuary, resulting in urban migration and resultant population growth. Consequently, there is an increase in the volume of industrial, domestic and municipal waste generated and discharged into the water system. Studies on of the QIRE and associated creeks were basically on the concentrations of trace metals and PAHs. However no data is available on the modeling of PAHs measured on the water system. This study therefore seeks to determine the contamination status, distribution and source apportionment of sixteen priority PAHs in the QIRE system.

\section{Materials and Methods}

\subsection{The Study Area and Site Description}

Qua Iboe river is one of the major rivers in the Niger Delta region of Nigeria. It originates from a waterfall in Umuahia hills and flows southwards across Umudike into Akwa Ibom state. Five sampling sites are located at the lower reach of Qua Iboe River in Ibeno local government area close to a petrochemical effluent treatment and discharge plant while the control site is located at Ekpene Ukpa in Etinan local government area of Akwalbom State, about $27 \mathrm{~km}$ from the examined sites and is free from oil exploration and production activities. Ibeno lies on the eastern side of Qua Iboe River about $3 \mathrm{~km}$ from the river and is one of the largest fishing settlements in the Nigerian coast. The global positioning system (GPS) coordinates of the sampling sites are: Okoroutip $\left(4^{\circ} 55^{\prime} 5^{\prime \prime} \mathrm{N}-7^{\circ} 54^{\prime} 47^{\prime \prime} \mathrm{E}\right)$, Ukpenekang

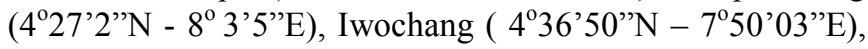
Douglas creek $\left(4^{\circ} 30^{\prime} 55^{\prime} \mathrm{N}-8^{\circ} 07^{\prime} \mathrm{E}\right)$, Stubb creek $\left(4^{\circ} 34^{\prime} 41^{\prime \prime} \mathrm{N}-7^{\circ} 59^{\prime} 47^{\prime \prime} \mathrm{E}\right), \quad$ EkpeneUkpa $\left(4^{\circ} 47^{\prime} 90^{\prime \prime} \mathrm{N}-\right.$ $\left.7^{\circ} 50^{\prime} 03^{\prime \prime} \mathrm{E}\right)$. Figure 1 is a map of the study area indicating the sampling sites.

\subsection{Samples and Sampling}

Sample containers and glassware for PAH analyses were rinsed with hexane and dichloromethane to remove adhering polar and non-polar compounds [11]. Surface water samples for the analysis of PAHs were collected using an ambercoloured borosilicate 1 litre capacity glass bottles with teflonlined caps to prevent photochemical degradation and 3 drops of $1 \mathrm{M} \mathrm{HCl}$ was added to prevent bacterial degradation of the sample respectively [12]. Sampling was conducted monthly from November, 2013 to October, 2014. Sub-samples from five points per sampling site were homogenized to form a composite sample. A total of three hundred and sixty subsamples and 72 composite samples were collected.

Extraction and Clean up

The extraction of the samples was carried out by liquidliquid extraction method protocol described by [13] and the clean-up was carried out in a column chromatography packed with $\mathrm{Na}_{2} \mathrm{SO}_{4}$ and silica gel.

\subsection{Determination of PAHs Concentration}

The concentration of PAHs was determined using standard protocol described by [12]. The sample was automatically detected as it emerged from the column by a flame ionization detector (FID) by measuring the retention time. The identification of the PAH compounds was achieved using Chemstation software and was based on matching their retention time with calibrated PAH standards while quantification was obtained from the corresponding areas of the respective chromatograms. Procedural blanks and solvent blanks were analysed and quantified with no PAHs found in these blanks. Prior to use, the GC was calibrated using a five point calibration curve established using dichloromethanebased standards (Accustandard PAH mix, $1000 \mu \mathrm{g} / \mathrm{ml}$ in $\left.\mathrm{CH}_{2} \mathrm{Cl}_{2}\right)$. The coefficient of determination values $\left(\mathrm{R}^{2}\right)$ were greater than 0.87 . Prior to extraction, four surrogate standards were added to the sample to take care of unusual matrix effect. These internal surrogate standards were acenaphthene$\mathrm{d}_{10}$, phenanthrene-d10, chrysene-d12 and perylene-d12 from Smart solutions, USA.

\section{Chromatographic Conditions}

The gas chromatograph was Agilent Hewlett Packard 5890 series II, coupled with flame ionization detector (FID) powered with HP Chemstation Rev. A. 0901 (10206) software. To identify and quantify PAH components, the following GC operating conditions was utilized:

Detector: Hydrogen at $35 \mathrm{ml} / \mathrm{min}$; air at $250 \mathrm{ml} / \mathrm{mins}$ and nitrogen at $30 \mathrm{ml} / \mathrm{min}$.

Oven: Initial temperature $65^{\circ} \mathrm{C}$; final temperature; $325^{\circ} \mathrm{C}$, Run time: 30 minute

Inlet: Splitless injection was adopted using a rubber septum and the volume injected was $1 \mu 1$. The inlet temperature was $275^{\circ} \mathrm{C}$, with a pressure of 14.8 Psi and total flow of $65.4 \mathrm{ml} / \mathrm{min}$.

Column: The column was lined with 1,3-dimethyl polysiloxane with capillary HPS type of $30 \mathrm{~m}$ length, $0.32 \mathrm{~mm}$ wide bore diameter, $0.25 \mu \mathrm{m}$ film diameter.

\subsection{Identification of PAH Sources}

Diagnostic ratios were used to identify the possible sources of PAH in the water bodies. The following ratios were used as source indicators: Fluoranthene/Fluoranthene + Pyrene ratio (Flue / Flue + Pyr), Anthracene/Anthracene + Phenanthrene ratio (Ant / Ant + Phe) and LMW-PAH to HMW-PAH ratio. 


\subsection{Statistical Analysis}

All values were expressed as mean of six determinations \pm standard deviation. Student's t-test was used to compare between the mean of total PAH values in both the dry and wet seasons and a $\mathrm{P}<0.05$ was considered statistically significant. Pearson correlation coefficient was used to examine the relationship between PAH pairs in water and cluster analysis was used to assess common pollution sources between sampling sites. Statistical analyses were performed using SPSS statistics 17.0 windows.

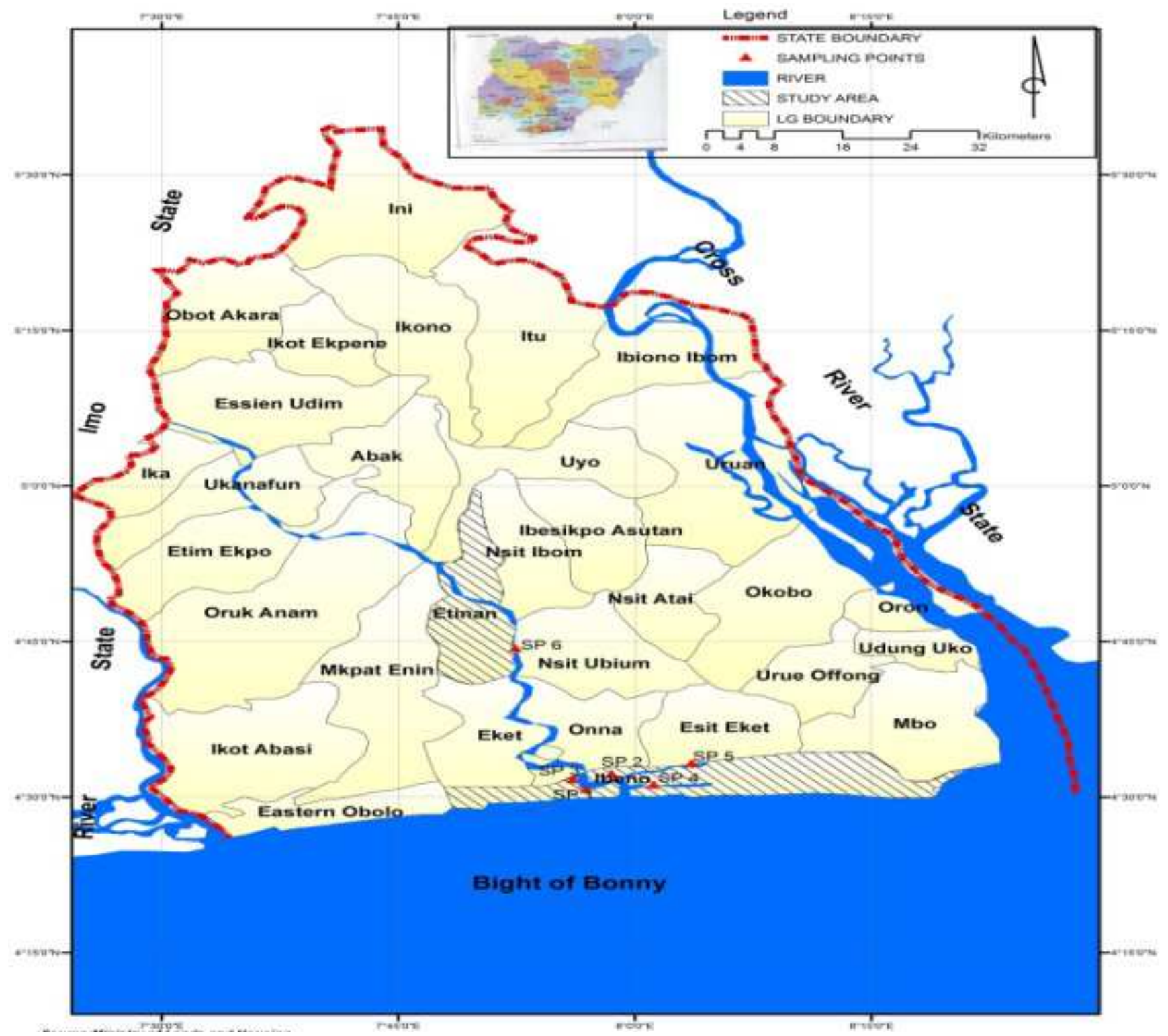

Figure 1. It is a map of the study area indicating the sampling sites.

\section{Results and Discussion}

Concentration of the 16 priority PAHs in water from QIRE in both dry and wet seasons are shown in Tables 1, 2, 3 , and 4 . The mean total PAHs ranged from 7.827E-04 for the wet season to $1.500 \mathrm{E}-02 \mathrm{mg} / 1$ for the dry season. Results revealed significantly higher $(\mathrm{P}<0.05) \quad \mathrm{PAH}$ concentration in the wet season, compared to the dry season. In addition mean PAH concentrations were significantly higher $(\mathrm{P}<0.05)$ at the sampled sites than the control. In the dry and wet seasons, the total PAHs for the water sample at all the sampling sites were above the permissible limit except at the control site. The high levels of PAHs in water at the examined sites may be due to atmospheric fall-out from continuous gas flaring and waste water discharge from a petrochemical plant situated close to the two creeks. Studies by [14] showed that the pathways for PAHs to enter surface water include atmospheric fallout, urban-run off, municipal effluents, sewage discharge, industrial effluents and oil spillage while High levels of $\mathrm{B}(\mathrm{k}) \mathrm{F}$ and $\operatorname{lnd}(1,2,3-\mathrm{cd}) \mathrm{P}$ in water from the Niger Delta were linked to run-off from petroleum by-products (4). Higher PAH values recorded in the wet season compared to the dry season may be due to flooding, possible pipeline 
vandalisation and oil spillage that occurred in the month of July during the sampling period [15]. The level of individual PAHs from the present study were lower than levels reported for Cankiri, Turkey [16], but comparable to levels in other areas of the Niger Delta [4, 17].The occurrence of low molecular weight PAHs (LMW- PAHs) ranged from $0.49 \%$ to $22.7 \%$ in the dry season and $0.07 \%$ to $3.67 \%$ in the wet season. The occurrence of High molecular weight-PAHs (HMW-PAHs) ranged from $77.1 \%$ to $99.5 \%$ in the dry season and $98.6 \%$ to $99.9 \%$ in the wet season. Low percentage of low molecular weight PAHs (LMWPAHs) compared with high molecular weight PAHs (HMW$\mathrm{PAH})$ was observed in this study. The low percentage of LMW PAHs may be due to their possible volatilization to the atmospheredue to their relatively high vapour pressure. Also, 2-3 ring PAHs are more easily biodegraded and volatilized compared with 4-6 ring PAHs. Moreover, the higher levels of HMW-PAHs in this study may be due to their attachment on soot produced from continuous gas flaring from an oil exploration industry which eventually deposits into the water [18]. The Findings from this study are different from the findings of other studies with reports of dominance of 2-3 rings PAHs [19, 20]. The differences observed from other studies were attributed to differences in anthropogenic inputs of PAHs to the water bodies. For instance, PAHs in estuarine water from Wenzou, China were sourced from leather industries.

Table 1. Concentration of low molecular weight polycyclic aromatic hydrocarbon with fluoranthene and pyrene in water from QIRE in the dry Season.

\begin{tabular}{|c|c|c|c|c|c|c|c|}
\hline PAH mixture & & Okoroutip & Ukpenekang & Iwochang & Douglas & Stubb & EkpeneUkpa \\
\hline \multirow{2}{*}{ Naphthalene } & Mean & $\begin{array}{l}1.639 \mathrm{E}-04 \pm \\
4.327 \mathrm{E}-05\end{array}$ & $\begin{array}{l}1.025 \mathrm{E}-04 \pm \\
1.522 \mathrm{E}-05\end{array}$ & $\begin{array}{l}2.213 \mathrm{E}-04 \pm \\
2.828-06\end{array}$ & $\begin{array}{l}2.055 \mathrm{E}-04 \pm \\
5.03-05\end{array}$ & $\begin{array}{l}6.730 \mathrm{E}-05 \pm \\
2.081-06\end{array}$ & $\begin{array}{l}8.9135 \mathrm{E}-05 \pm \\
1.5044-06\end{array}$ \\
\hline & Range & $\begin{array}{l}1.333 \mathrm{E}-04 \text { to } \\
1.945 \mathrm{E}-04\end{array}$ & $\begin{array}{l}9.180 \mathrm{E}-05 \text { to } \\
1.130 \mathrm{E}-04\end{array}$ & $\begin{array}{l}1.326 \mathrm{E}-04 \text { to } \\
3.100 \mathrm{E}-04\end{array}$ & $\begin{array}{l}1.287 \mathrm{E}-04 \text { to } \\
2.824 \mathrm{E}-04\end{array}$ & $\begin{array}{l}5.604 \mathrm{E}-05 \text { to } \\
7.803 \mathrm{E}-05\end{array}$ & $\begin{array}{l}8.642 \mathrm{E}-05 \text { to } \\
9.85 \mathrm{E}-05\end{array}$ \\
\hline Acenaphthylene & Mean & $\begin{array}{l}1.779 \mathrm{E}-06 \pm \\
3.7260 \mathrm{E}-07\end{array}$ & $\begin{array}{l}5.3175 \mathrm{E}-06 \pm \\
2.560 \mathrm{E}-07\end{array}$ & $\begin{array}{l}2.006 \mathrm{E}-06 \pm \\
2.000-08\end{array}$ & $\begin{array}{l}4.632 \mathrm{E}-06 \pm \\
4.163-07\end{array}$ & $\begin{array}{l}1.868 \mathrm{E}-05 \pm \\
2.516-06\end{array}$ & $\begin{array}{l}2.337 \mathrm{E}-06 \pm \\
1.958-07\end{array}$ \\
\hline \multirow{2}{*}{ Acenaphthene } & Mean & $\begin{array}{l}6.3457 \mathrm{E}-06 \pm \\
4.979 \mathrm{E}-07\end{array}$ & $\begin{array}{l}3.2945 \mathrm{E}-06 \pm \\
5.820 \mathrm{E}-08\end{array}$ & $\begin{array}{l}4.6215 \mathrm{E}-07 \pm \\
3.511-09\end{array}$ & $\begin{array}{l}2.785 \mathrm{E}-06 \pm \\
2.00-07\end{array}$ & $\begin{array}{l}3.2561 \mathrm{E}-06 \pm \\
2.413-07\end{array}$ & $\begin{array}{l}2.552 \mathrm{E}-06 \pm \\
3.964-08\end{array}$ \\
\hline & Range & $\begin{array}{l}2.186 \mathrm{E}-06 \text { to } \\
1.323 \mathrm{E}-05\end{array}$ & $\begin{array}{l}2.359 \mathrm{E}-06 \text { to } \\
4.230 \mathrm{E}-06\end{array}$ & $\begin{array}{l}2.253 \mathrm{E}-07 \text { to } \\
6.990 \mathrm{E}-07\end{array}$ & $\begin{array}{l}\text { N.D. to } \\
1.114 \mathrm{E}-06\end{array}$ & $\begin{array}{l}6.950 \mathrm{E}-07 \text { to } \\
7.257 \mathrm{E}-06\end{array}$ & $\begin{array}{l}3.250 \mathrm{E}-06 \text { to } \\
4.779 \mathrm{E}-06\end{array}$ \\
\hline Fluorene & Mean & $\begin{array}{l}6.8507 \mathrm{E}-06 \pm \\
1.7039 \mathrm{E}-07\end{array}$ & $\begin{array}{l}6.372 \mathrm{E}-05 \pm \\
1.12 \mathrm{E}-06\end{array}$ & $\begin{array}{l}5.589 \mathrm{E}-05 \pm \\
4.041 \mathrm{E}-06\end{array}$ & $\begin{array}{l}1.2507 \mathrm{E}-05 \pm \\
2.0816-07\end{array}$ & $\begin{array}{l}1.5902 \mathrm{E}-04 \pm \\
3.1-06\end{array}$ & $\begin{array}{l}6.823 \mathrm{E}-05 \pm \\
2.431-06\end{array}$ \\
\hline \multirow{2}{*}{ Phenanthrene } & Mean & $\begin{array}{l}3.774 \mathrm{E}-05 \pm \\
6.185-06\end{array}$ & $\begin{array}{l}3.006 \mathrm{E}-05 \pm \\
5.781-06\end{array}$ & $\begin{array}{l}5.872 \mathrm{E}-05 \pm \\
1.634-06\end{array}$ & $\begin{array}{l}6.076 \mathrm{E}-05 \pm \\
4.505-07\end{array}$ & $\begin{array}{l}2.608 \mathrm{E}-05 \pm \\
2.00 \mathrm{E}-07\end{array}$ & $\begin{array}{l}9.3975 \mathrm{E}-06 \pm \\
2.081 \mathrm{E}-08\end{array}$ \\
\hline & Range & $\begin{array}{l}1.066 \mathrm{E}-06 \text { to } \\
1.388 \mathrm{E}-04\end{array}$ & $\begin{array}{l}2.741 \mathrm{E}-06 \text { to } \\
6.452 \mathrm{E}-05\end{array}$ & $\begin{array}{l}1.548 \mathrm{E}-06 \text { to } \\
1.159 \mathrm{E}-04\end{array}$ & $\begin{array}{l}1.002 \mathrm{E}-05 \text { to } \\
1.115 \mathrm{E}-04\end{array}$ & $\begin{array}{l}1.391 \mathrm{E}-06 \text { to } \\
7.361 \mathrm{E}-05\end{array}$ & $\begin{array}{l}7.2225 \mathrm{E}-06 \text { to } \\
1.157 \mathrm{E}-05\end{array}$ \\
\hline \multirow[t]{2}{*}{ Anthracene } & Mean & $\begin{array}{l}3.714 \mathrm{E}-05 \pm \\
8.976 \mathrm{E}-07\end{array}$ & $\begin{array}{l}2.618 \mathrm{E}-05 \pm \\
7.919 \mathrm{E}-08\end{array}$ & $\begin{array}{l}8.5325 \mathrm{E}-06 \pm \\
2.641 \mathrm{E}-07\end{array}$ & $\begin{array}{l}9.042 \mathrm{E}-06 \pm \\
2.645 \mathrm{E}-08\end{array}$ & $\begin{array}{l}3.0550 \mathrm{E}-08 \pm \\
3.0550 \mathrm{E}-08\end{array}$ & $\begin{array}{l}3.655 \mathrm{E}-06 \pm \\
4.321 \mathrm{E}-07\end{array}$ \\
\hline & Range & $\begin{array}{l}3.618 \mathrm{E}-06 \text { to } \\
4.176 \mathrm{E}-06\end{array}$ & $\begin{array}{l}2.562 \mathrm{E}-06 \text { to } \\
2.674 \mathrm{E}-06\end{array}$ & $\begin{array}{l}4.145 \mathrm{E}-06 \text { to } \\
1.292 \mathrm{E}-05\end{array}$ & $\begin{array}{l}3.325 \mathrm{E}-06 \text { to } \\
1.476 \mathrm{E}-05\end{array}$ & $\begin{array}{l}5.981 \mathrm{E}-06 \text { to } \\
4.500 \mathrm{E}-06\end{array}$ & $\begin{array}{l}6.035 \mathrm{E}-08 \text { to } \\
1.057 \mathrm{E}-05\end{array}$ \\
\hline \multirow{2}{*}{ Fluoranthene } & Mean & $\begin{array}{l}2.250 \mathrm{E}-04 \pm \\
8485 \mathrm{E}-05\end{array}$ & $\begin{array}{l}3.902 \mathrm{E}-04 \pm \\
1.760 \mathrm{E}-05\end{array}$ & $\begin{array}{l}1.084 \mathrm{E}-05 \pm \\
1.527 \mathrm{E}-06\end{array}$ & $\begin{array}{l}1.969 \mathrm{E}-04 \pm \\
4.932 \mathrm{E}-06\end{array}$ & $\begin{array}{l}9.939 \mathrm{E}-05 \pm \\
2.00 \mathrm{E}-06\end{array}$ & $\begin{array}{l}1.443 \mathrm{E}-04 \pm \\
3.911 \mathrm{E}-06\end{array}$ \\
\hline & Range & $\begin{array}{l}2.904 \mathrm{E}-06 \text { to } \\
6.260 \mathrm{E}-04\end{array}$ & $\begin{array}{l}6.219 \mathrm{E}-06 \text { to } \\
6.290 \mathrm{E}-04\end{array}$ & $\begin{array}{l}3.456 \mathrm{E}-06 \text { to } \\
3.200 \mathrm{E}-04\end{array}$ & $\begin{array}{l}7.325 \mathrm{E}-05 \text { to } \\
3.206 \mathrm{E}-04\end{array}$ & $\begin{array}{l}2.821 \mathrm{E}-06 \text { to } \\
2.911 \mathrm{E}-04\end{array}$ & $\begin{array}{l}6.325 \mathrm{E}-07 \text { to } \\
2.88 \mathrm{E}-04\end{array}$ \\
\hline \multirow{2}{*}{ Pyrene } & Mean & $\begin{array}{l}1.525 \mathrm{E}-05 \pm \\
7.767 \mathrm{E}-07\end{array}$ & $\begin{array}{l}1.904 \mathrm{E}-06 \pm \\
7.735 \mathrm{E}-08\end{array}$ & $\begin{array}{l}7.571 \mathrm{E}-04 \pm \\
5.131 \mathrm{E}-05\end{array}$ & $\begin{array}{l}8.758 \mathrm{E}-04 \pm \\
3.963 \mathrm{E}-05\end{array}$ & $\begin{array}{l}8.784 \mathrm{E}-04 \pm \\
2.532 \mathrm{E}-05\end{array}$ & $\begin{array}{l}8.221 \mathrm{E}-05 \pm \\
2.516 \mathrm{E}-07\end{array}$ \\
\hline & Range & $\begin{array}{l}6.474 \mathrm{E}-06 \text { to } \\
2.676 \mathrm{E}-05\end{array}$ & $\begin{array}{l}1.119 \mathrm{E}-06 \text { to } \\
3.265 \mathrm{E}-06\end{array}$ & $\begin{array}{l}3.224 \mathrm{E}-06 \text { to } \\
2.216 \mathrm{E}-03\end{array}$ & $\begin{array}{l}1.199 \mathrm{E}-04 \text { to } \\
2.155 \mathrm{E}-03\end{array}$ & $\begin{array}{l}3.855 \mathrm{E}-06 \text { to } \\
2.578 \mathrm{E}-03\end{array}$ & $\begin{array}{l}7.325 \mathrm{E}-07 \text { to } \\
1.637 \mathrm{E}-04\end{array}$ \\
\hline
\end{tabular}

Results are presented as Mean \pm SD

Table 2. Concentration of high molecular weight polycyclic aromatic hydrocarbon in water from QIRE in the dry Season.

\begin{tabular}{|c|c|c|c|c|c|c|c|}
\hline $\begin{array}{l}\text { PAH } \\
\text { mixture }\end{array}$ & & Okoroutip & Ukpenekang & Iwochang & Douglas & Stubb & EkpeneUkpa \\
\hline \multirow{4}{*}{$\mathrm{B}(\mathrm{a}) \mathrm{A}$} & \multirow{2}{*}{ Mean } & $6.454 \mathrm{E}-06 \pm$ & 8.377E-05士 & 1.333E-05士 & 8.580E-06士 & 5.603E-06士 & $1.8205 \mathrm{E}-06 \pm$ \\
\hline & & $6.807 \mathrm{E}-07$ & $2.347 \mathrm{E}-06$ & $3.055 \mathrm{E}-07$ & $3.577 \mathrm{E}-07$ & $2.594 \mathrm{E}-07$ & $3.2145 \mathrm{E}-08$ \\
\hline & \multirow{2}{*}{ Range } & $5.340 \mathrm{E}-07$ to & $8.211 \mathrm{E}-05$ to & $6.336 \mathrm{E}-06$ to & $7.325 \mathrm{E}-06$ to & $1.286 \mathrm{E}-06$ to & $2.281 \mathrm{E}-07$ to \\
\hline & & $1.291 \mathrm{E}-05$ & $8.543 \mathrm{E}-05$ & $2.033 \mathrm{E}-05$ & $9.836 \mathrm{E}-06$ & $1.320 \mathrm{E}-05$ & $3.413 \mathrm{E}-06$ \\
\hline \multirow{3}{*}{ Chysene } & \multirow{2}{*}{ Mean } & 3.445E-05士 & $1.8293 \mathrm{E}-05 \pm$ & 5.790E-05士 & $3.260 \mathrm{E}-05 \pm$ & $1.824 \mathrm{E}-05 \pm$ & $7.8973 \mathrm{E}-06 \pm$ \\
\hline & & $4.582 \mathrm{E}-06$ & $1.7785 \mathrm{E}-07$ & 2.902E-06 & $2.503 \mathrm{E}-06$ & $3.214 \mathrm{E}-07$ & $2.523 \mathrm{E}-07$ \\
\hline & Range & $1.253 \mathrm{E}-04$ & $2.041 \mathrm{E}-05$ & $1.091 \mathrm{E}-04$ & 8.199E-05 & 4.767E-05 & $1.536 \mathrm{E}-05$ \\
\hline
\end{tabular}




\begin{tabular}{|c|c|c|c|c|c|c|c|}
\hline $\begin{array}{l}\text { PAH } \\
\text { mixture }\end{array}$ & & Okoroutip & Ukpenekang & Iwochang & Douglas & Stubb & EkpeneUkpa \\
\hline \multirow{4}{*}{$\mathrm{B}(\mathrm{b}) \mathrm{F}$} & \multirow{2}{*}{ Mean } & $4.589 \mathrm{E}-03 \pm$ & $1.368 \mathrm{E}-03 \pm$ & $1.975 \mathrm{E}-02 \pm$ & $3.267 \mathrm{E}-02 \pm$ & $1.111 \mathrm{E}-02 \pm$ & $3.0118 \mathrm{E}-04 \pm$ \\
\hline & & $3.732 \mathrm{E}-04$ & 4.46E-04 & $2.081 \mathrm{E}-03$ & $1.552 \mathrm{E}-03$ & $2.289 \mathrm{E}-04$ & $4.509 \mathrm{E}-06$ \\
\hline & \multirow{2}{*}{ Range } & $2.187 \mathrm{E}-04$ to & $1.699 \mathrm{E}-04$ to & $3.652 \mathrm{E}-04$ to & $1.429 \mathrm{E}-04$ to & $4.347 \mathrm{E}-05$ to & $7.001 \mathrm{E}-05$ to \\
\hline & & $1.565 \mathrm{E}-02$ & $1.882 \mathrm{E}-03$ & $5.462 \mathrm{E}-02$ & $3.542 \mathrm{E}-02$ & $2.614 \mathrm{E}-02$ & $4.236 \mathrm{E}-04$ \\
\hline \multirow{4}{*}{$\mathrm{B}(\mathrm{k}) \mathrm{F}$} & \multirow{2}{*}{ Mean } & 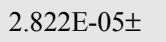 & 4.303E-05 \pm & $3.011 \mathrm{E}-04 \pm$ & 2.503E-05 \pm & 4.552E-05 \pm & $3.5312 \mathrm{E}-06 \pm$ \\
\hline & & $3.214 \mathrm{E}-07$ & $8.695 \mathrm{E}-07$ & $1.518 \mathrm{E}-05$ & $2.383 \mathrm{E}-06$ & 052E-04 & $4.875 \mathrm{E}-08$ \\
\hline & \multirow{2}{*}{ Range } & $3.461 \mathrm{E}-07$ to & $3.962 \mathrm{E}-06$ to & $2.362 \mathrm{E}-06$ to & $2.932 \mathrm{E}-07$ to & $3.652 \mathrm{E}-05$ to & $3.645 \mathrm{E}-07$ to \\
\hline & & $1.009 \mathrm{E}-04$ & $1.199 \mathrm{E}-04$ & $3652 \mathrm{E}-04$ & 7.146E-05 & $1.247 \mathrm{E}-04$ & $6.698 \mathrm{E}-06$ \\
\hline \multirow{4}{*}{$\mathrm{B}(\mathrm{a}) \mathrm{P}$} & \multirow{2}{*}{ Mean } & $1.9488 \mathrm{E}-05 \pm$ & $2.945 \mathrm{E}-05 \pm$ & $1.376 \mathrm{E}-05 \pm$ & 2.354E-05士 & $2.342 \mathrm{E}-05 \pm$ & $4.1585 \mathrm{E}-06 \pm$ \\
\hline & & 4.061E-06 & $1.603 \mathrm{E}-06$ & $2.013 \mathrm{E}-07$ & $2.511 \mathrm{E}-07$ & $5.312 \mathrm{E}-06$ & $2.865 \mathrm{E}-07$ \\
\hline & \multirow{2}{*}{ Range } & $4.532 \mathrm{E}-06$ to & $3.365 \mathrm{E}-06$ to & $8.669 \mathrm{E}-06$ to & $4.733 \mathrm{E}-07$ to & $7.965 \mathrm{E}-06$ to & $1.890 \mathrm{E}-05$ to \\
\hline & & $3.250 \mathrm{E}-04$ & $5.261 \mathrm{E}-05$ & $2.001 \mathrm{E}-05$ & $6.914 \mathrm{E}-05$ & $4.263 \mathrm{E}-05$ & $6.421 \mathrm{E}-06$ \\
\hline \multirow{4}{*}{$\begin{array}{l}\text { Lnd } \\
(1,2,3, \mathrm{~cd}) \mathrm{P}\end{array}$} & \multirow{2}{*}{ Mean } & $3.931 \mathrm{E}-03 \pm$ & $2.949 \mathrm{E}-05 \pm$ & $1.1634 \mathrm{E}-02 \pm$ & $2.380 \mathrm{E}-03 \pm$ & $1.9921 \mathrm{E}-03 \pm$ & 4.427E-05士 \\
\hline & & $4.061 \mathrm{E}-05$ & $1.603 \mathrm{E}-06$ & 7.29E-03 & $3.04 \mathrm{E}-05$ & $1.111 \mathrm{E}-04$ & $8.821 \mathrm{E}-07$ \\
\hline & \multirow{2}{*}{ Range } & $2.077 \mathrm{E}-03$ to & $2.350 \mathrm{E}-04$ to & $2.256 \mathrm{E}-05$ to & $3.117 \mathrm{E}-04$ to & $8.555 \mathrm{E}-05$ to & $8.321 \mathrm{E}-07$ to \\
\hline & & $7.256 \mathrm{E}-03$ & $1.967 \mathrm{E}-02$ & $1.237 \mathrm{E}-03$ & $3.506 \mathrm{E}-03$ & $2.567 \mathrm{E}-03$ & $8.772 \mathrm{E}-05$ \\
\hline \multirow{4}{*}{$\mathrm{D}(\mathrm{ah}) \mathrm{A}$} & \multirow{2}{*}{ Mean } & $4.188 \mathrm{E}-06 \pm$ & $2.830 \mathrm{E}-03 \pm$ & $4.3416 \mathrm{E}-05 \pm$ & $3.922 \mathrm{E}-05 \pm$ & $5.6102 \mathrm{E}-05 \pm$ & $5.4545 \mathrm{E}-06 \pm$ \\
\hline & & $5.519 \mathrm{E}-07$ & $3.785 \mathrm{E}-05$ & $2.081 \mathrm{E}-07$ & $1.0094 \mathrm{E}-06$ & $1.6258 \mathrm{E}-07$ & $8.5040 \mathrm{E}-07$ \\
\hline & \multirow{2}{*}{ Range } & $3.267 \mathrm{E}-06$ to & $2.826 \mathrm{E}-05$ to & $1.901 \mathrm{E}-05$ to & $5.245 \mathrm{E}-07$ to & $6.474 \mathrm{E}-07$ to & $3.583 \mathrm{E}-06$ to \\
\hline & & $9.735 \mathrm{E}-06$ & $8.401 \mathrm{E}-03$ & 7.161E-05 & $4.352 \mathrm{E}-05$ & $1.143 \mathrm{E}-04$ & 7.326E-06 \\
\hline \multirow{4}{*}{ B(ghl)P } & \multirow{2}{*}{ Mean } & 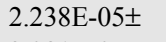 & $1.376 \mathrm{E}-05 \pm$ & 4.687E-05士 & $1.471 \mathrm{E}-03 \pm$ & $5.327 \mathrm{E}-05 \pm$ & $1.724 \mathrm{E}-04 \pm$ \\
\hline & & $2.081 \mathrm{E}-07$ & $6.021 \mathrm{E}-04$ & $1.907 \mathrm{E}-06$ & $4.006 \mathrm{E}-05$ & $3.399 \mathrm{E}-06$ & $8.934 \mathrm{E}-05$ \\
\hline & \multirow{2}{*}{ Range } & $1.862 \mathrm{E}-06$ to & 7.677E-05 to & $4.068 \mathrm{E}-06$ to & $3.815 \mathrm{E}-07$ to & $6.6638 \mathrm{E}-07$ to & $1.724 \mathrm{E}-04$ to \\
\hline & & $4.690 \mathrm{E}-05$ & $1.307 \mathrm{E}-04$ & $9.630 \mathrm{E}-05$ & 4.076E-03 & $8.603 \mathrm{E}-05$ & 8.934E-05 \\
\hline
\end{tabular}

Results are presented as Mean $\pm \mathrm{SD}$

Note: $\mathrm{B}(\mathrm{a}) \mathrm{A}=\mathrm{Benzo}(\mathrm{a})$ anthracene, $\mathrm{B}(\mathrm{b}) \mathrm{F}=\mathrm{Benzo}(\mathrm{b})$ Fluoranthene, $\mathrm{B}(\mathrm{k}) \mathrm{F}=$ Benzo(k)Fluoranthene, $\mathrm{B}(\mathrm{a}) \mathrm{P}=$ Benzo (a) Pyrene,

Ind (1,2,3-cd)P = Indeno(1,2,3-cd)Pyrene, D(ah)A = Dibenzo(a,h) Anthracene, B(ghi)P = Benzo(ghi)Perylene

Table 3. Concentration of low molecular weight polycyclic aromatic hydrocarbon with fluoranthene and pyrene in water from QIRE in the wet Season.

\begin{tabular}{|c|c|c|c|c|c|c|c|}
\hline PAH mixture & & Okoroutip & Ukpenekang & Iwochang & Douglas & Stubb & EkpeneUkpa \\
\hline \multirow{3}{*}{ Naphthalene } & Mean & $\begin{array}{l}6.116 \mathrm{E}-06 \pm \\
3.0 \mathrm{E}-07\end{array}$ & $\begin{array}{l}6.5417 \mathrm{E}-06 \pm \\
1.123 \mathrm{E}-07\end{array}$ & $\begin{array}{l}2.211 \mathrm{E}-05 \pm \\
3.324 \mathrm{E}-06\end{array}$ & $\begin{array}{l}1.849 \mathrm{E}-04 \pm \\
3.6055 \mathrm{E}-06\end{array}$ & $\begin{array}{l}9.987 \mathrm{E}-06 \pm \\
6.245 \mathrm{E}-08\end{array}$ & $\begin{array}{l}1.581 \mathrm{E}-07 \pm \\
2.2 \mathrm{E}-09\end{array}$ \\
\hline & \multirow{2}{*}{ Range } & $2.252 \mathrm{E}-06$ to & $2.532 \mathrm{E}-07$ to & $1.6204 \mathrm{E}-06$ to & $2.416 \mathrm{E}-06$ to & $6.352 \mathrm{E}-05$ to & $2.963 \mathrm{E}-05$ to \\
\hline & & $1.236 \mathrm{E}-05$ & $1.806 \mathrm{E}-05$ & $0.681 \mathrm{E}-04$ & $5.216 \mathrm{E}-04$ & $1.230 \mathrm{E}-04$ & 3.392E-05 \\
\hline \multirow{4}{*}{ Acenaphthylene } & \multirow{2}{*}{ Mean } & $1.6544 \mathrm{E}-06 \pm$ & $2.236 \mathrm{E}-06 \pm$ & $2.041 \mathrm{E}-04 \pm$ & $1.261 \mathrm{E}-06 \pm$ & $1.4618 \mathrm{E}-05 \pm$ & 5.274E-08土 \\
\hline & & $3.511 \mathrm{E}-08$ & $2.0816 \mathrm{E}-08$ & $1.00 \mathrm{E}-06$ & $3.6055 \mathrm{E}-08$ & $3.605 \mathrm{E}-07$ & 2.972E-09 \\
\hline & \multirow{2}{*}{ Range } & $1.600 \mathrm{E}-06$ to & $1.312 \mathrm{E}-07$ to & $2.341 \mathrm{E}-06$ to & $3.562 \mathrm{E}-07$ to & $3.733 \mathrm{E}-06$ to & N.D to \\
\hline & & $1.600 \mathrm{E}-06$ & $3.087 \mathrm{E}-06$ & $6.097 \mathrm{E}-04$ & $1.528 \mathrm{E}-06$ & $3.54 \mathrm{E}-05$ & $2.111 \mathrm{E}-07$ \\
\hline \multirow{4}{*}{ Acenaphthene } & \multirow{2}{*}{ Mean } & 4.340E-06士 & $7.048 \mathrm{E}-06 \pm$ & $1.5781 \mathrm{E}-04 \pm$ & $6.7925 \mathrm{E}-06 \pm$ & $6.228 \mathrm{E}-05 \pm$ & $3.613 \mathrm{E}-07 \pm$ \\
\hline & & $3 \mathrm{E}-08$ & $2.0816 \mathrm{E}-08$ & $4.582 \mathrm{E}-06$ & $2 \mathrm{E}-08$ & $2.6457 \mathrm{E}-08$ & 5.9197E-08 \\
\hline & \multirow{2}{*}{ Range } & $1.256 \mathrm{E}-07$ to & $7.0483 \mathrm{E}-06$ to & $3.336 \mathrm{E}-07$ to & $1.008 \mathrm{E}-06$ to & $3.369 \mathrm{E}-06$ to & N.D to \\
\hline & & $1.219 \mathrm{E}-05$ & $7.090 \mathrm{E}-06$ & $4.726 \mathrm{E}-04$ & $2.215 \mathrm{E}-04$ & $1.207 \mathrm{E}-05$ & $1.445 \mathrm{E}-06$ \\
\hline \multirow{4}{*}{ Fluorene } & \multirow{2}{*}{ Mean } & $4.287 \mathrm{E}-06 \pm$ & $1.677 \mathrm{E}-05 \pm$ & $2.9237 \mathrm{E}-05 \pm$ & $2.135 \mathrm{E}-06 \pm$ & $2.246 \mathrm{E}-05 \pm$ & $1.134 \mathrm{E}-05 \pm$ \\
\hline & & 4.5092E-08 & 5.859E-07 & $3.869 \mathrm{E}-06$ & $5.033 \mathrm{E}-07$ & $5.131 \mathrm{E}-07$ & $3.131 \mathrm{E}-07$ \\
\hline & \multirow{2}{*}{ Range } & $8.772 \mathrm{E}-07$ to & $9.25 \mathrm{E}-07$ to & $7.331 \mathrm{E}-07$ to & $2.500 \mathrm{E}-07$ to & $7.056 \mathrm{E}-06$ to & N.D to \\
\hline & & $1.103 \mathrm{E}-05$ & $5.407 \mathrm{E}-05$ & $1.079 \mathrm{E}-04$ & $4.822 \mathrm{E}-05$ & $1.207 \mathrm{E}-05$ & 4.537E-05 \\
\hline \multirow{4}{*}{ Phananthene } & \multirow{2}{*}{ Mean } & $1.240 \mathrm{E}-06 \pm$ & $1.740 \mathrm{E}-6 \pm$ & $2.348 \mathrm{E}-05 \pm$ & $2.1169 \mathrm{E}-05 \pm$ & $9.4216 \mathrm{E}-05 \pm$ & $9.236 \mathrm{E}-06 \pm$ \\
\hline & & $3.055 \mathrm{E}-08$ & $2 \mathrm{E}-08$ & $1.1135 \mathrm{E}-06$ & $4.358 \mathrm{E}-07$ & 1.9139E-06 & $2.668 \mathrm{E}-08$ \\
\hline & \multirow{2}{*}{ Range } & $1.342 \mathrm{E}-07$ to & $1.136 \mathrm{E}-06$ to & $1.119 \mathrm{E}-06$ to & $2.066 \mathrm{E}-07$ to & $7.251 \mathrm{E}-07$ to & $7.112 \mathrm{E}-06$ to \\
\hline & & $2.951 \mathrm{E}-06$ & $2.916 \mathrm{E}-06$ & $6.329 \mathrm{E}-05$ & $1.116 \mathrm{E}-05$ & $3.261 \mathrm{E}-04$ & $2.983 \mathrm{E}-05$ \\
\hline \multirow{4}{*}{ Anthracene } & \multirow{2}{*}{ Mean } & 2.289E-06士 & $3.876 \mathrm{E}-06 \pm$ & $1.988 \mathrm{E}-05 \pm$ & $1.390 \mathrm{E}-06 \pm$ & 1.8714E-05士 & $2.403 \mathrm{E}-06 \pm$ \\
\hline & & $9.6090 \mathrm{E}-08$ & $7.5605 \mathrm{E}-08$ & $3.5118 \mathrm{E}-07$ & $2.345 \mathrm{E}-05$ & 7.767E-07 & $3.5118 \mathrm{E}-05$ \\
\hline & \multirow{2}{*}{ Range } & $1.441 \mathrm{E}-06$ to & $4.073 \mathrm{E}-07$ to & $6.041 \mathrm{E}-07$ to & $1.242 \mathrm{E}-05$ to & $1.012 \mathrm{E}-07$ to & $1.321 \mathrm{E}-07$ to \\
\hline & & $3.212 \mathrm{E}-06$ & $7.345 \mathrm{E}-06$ & $1.455 \mathrm{E}-05$ & $4.1633 \mathrm{E}-07$ & $7.251 \mathrm{E}-05$ & $5.231 \mathrm{E}-06$ \\
\hline \multirow{4}{*}{ Fluoranthene } & \multirow{2}{*}{ Mean } & $1.9135 \mathrm{E}-05 \pm$ & $8.211 \mathrm{E}-06 \pm$ & $6.2706 \mathrm{E}-06 \pm$ & $1.5300 \mathrm{E}-05 \pm$ & 4.417E-05士 & 4.778E-06士 \\
\hline & & $7.2111 \mathrm{E}-07$ & 5.957E-07 & $2.565 \mathrm{E}-07$ & $8.1247 \mathrm{E}-06$ & $5.514 \mathrm{E}-07$ & 2.3985E-07 \\
\hline & \multirow{2}{*}{ Range } & $6.055 \mathrm{E}-06$ to & $1.532 \mathrm{E}-07$ to & $2.307 \mathrm{E}-06$ to & $3.346 \mathrm{E}-07$ to & $1.112 \mathrm{E}-06$ to & $2.761 \mathrm{E}-06$ to \\
\hline & & $2.823 \mathrm{E}-05$ & $1.200 \mathrm{E}-06$ & $1.218 \mathrm{E}-05$ & $2.605 \mathrm{E}-05$ & $4.125 \mathrm{E}-05$ & $8.675 \mathrm{E}-06$ \\
\hline \multirow{4}{*}{ Pyrene } & \multirow{2}{*}{ Mean } & $6.820 \mathrm{E}-06 \pm$ & 3.929E-07士 & $1.4587 \mathrm{E}-05 \pm$ & $3.175 \mathrm{E}-06 \pm$ & $1.592 \mathrm{E}-06 \pm$ & $1.938 \mathrm{E}-06 \pm$ \\
\hline & & $3.417 \mathrm{E}-07$ & $1.5557 \mathrm{E}-08$ & $4.428 \mathrm{E}-07$ & $2.083 \mathrm{E}-07$ & $3.031 \mathrm{E}-07$ & 2.328E-07 \\
\hline & \multirow{2}{*}{ Range } & $7.659 \mathrm{E}-08$ to & $3.265 \mathrm{E}-08$ to & $6.523 \mathrm{E}-08$ to & $6.398 \mathrm{E}-07$ to & $6.445 \mathrm{E}-07$ to & $6.034 \mathrm{E}-07$ to \\
\hline & & $2.246 \mathrm{E}-05$ & $1.280 \mathrm{E}-06$ & $3.593 \mathrm{E}-05$ & $6.334 \mathrm{E}-06$ & $3.897 \mathrm{E}-06$ & $5.0344 \mathrm{E}-06$ \\
\hline
\end{tabular}

Results are presented as Mean $\pm \mathrm{SD}$ 
Table 4. Concentration of high molecular weight polycyclic aromatic hydrocarbon in water from QIRE in the wet Season.

\begin{tabular}{|c|c|c|c|c|c|c|c|}
\hline PAH mixture & & Okoroutip & Ukpenekang & Iwochang & Douglas & Stubb & EkpeneUkpa \\
\hline \multirow{3}{*}{$\mathrm{B}(\mathrm{a}) \mathrm{A}$} & \multirow{2}{*}{ Mean } & 1.793E-05士 & 1.715E-05士 & $8.888 \mathrm{E}-06 \pm$ & $1.0769 \mathrm{E}-05 \pm$ & $6.1503 \mathrm{E}-06 \pm$ & $1.411 \mathrm{E}-07 \pm$ \\
\hline & & $7.150 \mathrm{E}-07$ & $2.396 \mathrm{E}-06$ & $3.428 \mathrm{E}-07$ & $2.4542 \mathrm{E}-07$ & $1.0400 \mathrm{E}-07$ & $1.6623 \mathrm{E}-09$ \\
\hline & Range & $5.617 \mathrm{E}-05$ & $4.312 \mathrm{E}-05$ & $2.903 \mathrm{E}-05$ & $3.251 \mathrm{E}-05$ & $7.216 \mathrm{E}-06$ & $5.642 \mathrm{E}-07$ \\
\hline \multirow{4}{*}{ Chysene } & \multirow{2}{*}{ Mean } & 1.392E-05士 & 4.355E-06士 & 1.312E-05士 & $1.220 \mathrm{E}-06 \pm$ & $6.198 \mathrm{E}-06 \pm$ & $5.450 \mathrm{E}-07 \pm$ \\
\hline & & $5.686 \mathrm{E}-07$ & $2.318 \mathrm{E}-07$ & $4.956 \mathrm{E}-07$ & $2.645 \mathrm{E}-08$ & 4.0722E-07 & $3.3350 \mathrm{E}-08$ \\
\hline & \multirow{2}{*}{ Range } & $3.250 \mathrm{E}-06$ to & $2.456 \mathrm{E}-07$ to & $4.223 \mathrm{E}-07$ to & $2.063 \mathrm{E}-07$ to & $6.630 \mathrm{E}-06$ to & \\
\hline & & $4.201 \mathrm{E}-05$ & $1.047 \mathrm{E}-05$ & $1.540 \mathrm{E}-06$ & $1.48 \mathrm{E}-06$ & $4.0722 \mathrm{E}-07$ & \\
\hline \multirow{4}{*}{$\mathrm{B}(\mathrm{b}) \mathrm{F}$} & \multirow{2}{*}{ Mean } & $1.703 \mathrm{E}-02 \pm$ & $9.327 \mathrm{E}-03 \pm$ & $2.928 \mathrm{E}-02 \pm$ & $1.272 \mathrm{E}-02 \pm$ & $2.073 \mathrm{E}-02 \pm$ & $1.715 \mathrm{E}-06 \pm$ \\
\hline & & $3.723 \mathrm{E}-03$ & $3.400 \mathrm{E}-04$ & $1.250 \mathrm{E}-03$ & $1.527 \mathrm{E}-03$ & $1.760 \mathrm{E}-03$ & $4.450 \mathrm{E}-08$ \\
\hline & \multirow{2}{*}{ Range } & $7.56 \mathrm{E}-03$ to & $6.712 \mathrm{E}-04$ to & $1.128 \mathrm{E}-02$ to & $6.546 \mathrm{E}-03$ to & $1.857 \mathrm{E}-02$ to & N.D to \\
\hline & & $1.123 \mathrm{E}-02$ & $3.429 \mathrm{E}-03$ & $6.868 \mathrm{E}-03$ & $3.251 \mathrm{E}-02$ & $2.145 \mathrm{E}-02$ & $6.859 \mathrm{E}-06$ \\
\hline \multirow{3}{*}{$\mathrm{B}(\mathrm{k}) \mathrm{F}$} & \multirow{2}{*}{ Mean } & $3.260 \mathrm{E}-06 \pm$ & 7.774E-06士 & 2.781E-06士 & $2.354 \mathrm{E}-06 \pm$ & $9.975 \mathrm{E}-05 \pm$ & $1.715 \mathrm{E}-06 \pm$ \\
\hline & & $4.1186 \mathrm{E}-08$ & $1.6522 \mathrm{E}-07$ & $3.605 \mathrm{E}-08$ & 4.725E-08 & $2.516 \mathrm{E}-07$ & 4.450E-08 \\
\hline & Range & $2.16 \mathrm{E}-07$ to & $6.342 \mathrm{E}-06$ to & $2.135 \mathrm{E}-07$ to & $1.367 \mathrm{E}-07$ to & $3.162 \mathrm{E}-07$ to & N.D to \\
\hline \multirow{4}{*}{$\mathrm{B}(\mathrm{a}) \mathrm{P}$} & \multirow{2}{*}{ Mean } & $1.4950 \mathrm{E}-05 \pm$ & 8.398E-06士 & 2.391E-05土 & $1.2938 \mathrm{E}-05 \pm$ & $2.826 \mathrm{E}-05 \pm$ & $9.943 \mathrm{E}-07 \pm$ \\
\hline & & 4.387E-07 & $6.5574 \mathrm{E}-08$ & $5.8966 \mathrm{E}-07$ & $4.725 \mathrm{E}-07$ & $4.041 \mathrm{E}-07$ & $9.068 \mathrm{E}-06$ \\
\hline & \multirow{2}{*}{ Range } & $3.542 \mathrm{E}-06$ to & $6.321 \mathrm{E}-06$ to & 5.513E-06 to & $2.063 \mathrm{E}-07$ to & $1.126 \mathrm{E}-06$ to & $2.516 \mathrm{E}-06$ to \\
\hline & & $3.165 \mathrm{E}-05$ & $1.221 \mathrm{E}-05$ & $2.826 \mathrm{E}-05$ & $7.561 \mathrm{E}-06$ & $1.985 \mathrm{E}-05$ & $3.214 \mathrm{E}-07$ \\
\hline \multirow{4}{*}{$\begin{array}{l}\text { Lnd } \\
(1,2,3, \mathrm{~cd}) \mathrm{P}\end{array}$} & \multirow{2}{*}{ Mean } & 1.029E-02士 & $2.594 \mathrm{E}-02 \pm$ & 4.942E-03土 & $4.685 \mathrm{E}-02 \pm$ & $3.338 \mathrm{E}-02 \pm$ & $5.872 \mathrm{E}-04 \pm$ \\
\hline & & $1.527 \mathrm{E}-03$ & $7.00 \mathrm{E}-03$ & $3.785 \mathrm{E}-04$ & $3.605 \mathrm{E}-03$ & $4.358 \mathrm{E}-03$ & $7.571 \mathrm{E}-05$ \\
\hline & \multirow{2}{*}{ Range } & $3.245 \mathrm{E}-03$ to & $7.55 \mathrm{E}-03$ to & $2.531 \mathrm{E}-04$ to & $2.456 \mathrm{E}-03$ to & $1.746 \mathrm{E}-03$ to & $3.323 \mathrm{E}-05$ to \\
\hline & & $1.213 \mathrm{E}-02$ & $6.372 \mathrm{E}-02$ & $1.17 \mathrm{E}-02$ & $7.694 \mathrm{E}-02$ & $6.523 \mathrm{E}-02$ & $1.332 \mathrm{E}-03$ \\
\hline \multirow{4}{*}{ D(ah)A } & \multirow{2}{*}{ Mean } & $2.062 \mathrm{E}-05 \pm$ & $2.466 \mathrm{E}-05 \pm$ & $7.285 \mathrm{E}-06 \pm$ & $3.1473 \mathrm{E}-05 \pm$ & $2.598 \mathrm{E}-06 \pm$ & $2.4217 \mathrm{E}-06 \pm$ \\
\hline & & $7.0116 \mathrm{E}-07$ & 4.9328E-07 & $2.023 \mathrm{E}-07$ & 1.619E-06 & $6.00 \mathrm{E}-08$ & 5.2915E-08 \\
\hline & \multirow{2}{*}{ Range } & $2.145 \mathrm{E}-06$ to & $1.461 \mathrm{E}-06$ to & 4.763E-08 to & $3.112 \mathrm{E}-07$ to & $4.451 \mathrm{E}-07$ to & $3.115 \mathrm{E}-08$ to \\
\hline & & $7.467 \mathrm{E}-05$ & $1.325 \mathrm{E}-05$ & $1.078 \mathrm{E}-05$ & $9.217 \mathrm{E}-05$ & 5.259E-06 & $4.714 \mathrm{E}-06$ \\
\hline \multirow{3}{*}{$\mathrm{B}(\mathrm{ghl}) \mathrm{P}$} & \multirow{2}{*}{ Mean } & $1.842 \mathrm{E}-05 \pm$ & $3.451 \mathrm{E}-05 \pm$ & $2.3753 \mathrm{E}-05 \pm$ & $1.759 \mathrm{E}-06 \pm$ & $1.054 \mathrm{E}-06 \pm$ & $1.596 \mathrm{E}-05 \pm$ \\
\hline & & $3.6055 \mathrm{E}-07$ & $7.088 \mathrm{E}-06$ & $7.023 \mathrm{E}-07$ & $7.211 \mathrm{E}-08$ & $7.505 \mathrm{E}-07$ & $6.557 \mathrm{E}-07$ \\
\hline & Range & $4.256 \mathrm{E}-06$ to & $1.086 \mathrm{E}-05$ to & $2.116 \mathrm{E}-07$ to & $9.321 \mathrm{E}-07$ to & $8.321 \mathrm{E}-06$ to & $3.402 \mathrm{E}-06$ to \\
\hline
\end{tabular}

$\mathrm{PAH}$ diagnostic ratio is used for identifying pollution sources [21-23]. In this study, Ant / Ant + Phe, Flue / Flue + Pyr and $\sum \mathrm{LMW} / \sum \mathrm{HMW}$ ratio were used for the identification of pyrogenic and petrogenic origins of PAHs. For molar mass of 178, ratio of Ant / Ant + Phe less than 0.1 indicate petrogenic origin while ratios greater than 0.1 indicates pyrogenic origin. For molar mass 202, Flue / Flue + Pyr $>0.5$ indicates pyrogenic $\mathrm{PAH}$ origin and the ratio $<0.4$ indicate petrogenic origin. $\sum \mathrm{LMW} / \sum \mathrm{HMW}$ ratio less than 1 indicates pyrogenic origin while $\sum \mathrm{LMW} / \sum \mathrm{HMW}$ ratio greater than 1 indicates petrogenic source $[22,24]$. Table 5 reveals the result of diagnostic ratio for PAHs in water from QIRE during the dry and wet season. In the dry season, the diagnostic ratio used in this study reveals pyrogenic PAH sources except at Iwochang where Flue/Flue + Pyre ratio is less than 0.5 signifying petrogenic $\mathrm{PAH}$ source. In the wet season, pyrogenic PAH sources was also observed for all the stations except for Iwochang for Flue/Flue $+\mathrm{Pyr}$ and Douglas creek for Ant/Ant + Phe.

The predominance of combustion (pyrogenic source) showed that PAHs in water from the examined sites are from combustion sources. The combustion sources of PAH in the water were likely to predominantly originate from constant gas flaring from a petrochemical facility located close to the sampled stations. Other combustion sources may have been emissions from combustion of fuel from automobiles and engine boats. The PAHs detected were predominantly HMWPAHs and therefore may have entered the water bodied through atmospheric deposition. Petrogenic PAHs are emitted directly to a water body while pyrogenic PAHs are first emitted into the air and enters the water through atmospheric fall-out [24]. In addition, mixed sources of PAH observed in this study compares with the result obtained by $[25,26]$.

The result of the correlation analyses conducted among the concentration of individual PAHs in water samples to assess $\mathrm{PAH}$ interrelationship and their origin during the dry and wet seasons are presented in Tables 6 and 7. Strong positive significant correlation was observed between some individual PAHs in water during the dry season. B(a)A and D(ah)A showed the highest PAH association with correlation coefficient of 0.994 , followed by Ind(1,2,3-cd)P and chrysene with correlation coefficient of 0.942 and Ind(1,2,3-cd)P and $\mathrm{B}(\mathrm{b}) \mathrm{F}$ with correlation coefficient of 0.938 . They were significant at 0.01 levels. $\mathrm{B}(\mathrm{k}) \mathrm{F}$ and chrysene interrelated strongly at 0.05 levels.

During the wet season, significant positive correlation was observed between Ind(1,2,3-cd)P and $\mathrm{B}(\mathrm{a}) \mathrm{P}(\mathrm{r}=0.719)$ at 0.01 levels while some $\mathrm{PAH}$ pairs recorded significant positive correlation at 0.05 levels. They are : $\mathrm{B}(\mathrm{b}) \mathrm{F} /$ chrysene ( $\mathrm{r}=0.789), \mathrm{D}(\mathrm{ah}) \mathrm{A} / \mathrm{B}(\mathrm{a}) \mathrm{P}(\mathrm{r}=0.657), \mathrm{D}(\mathrm{ah}) \mathrm{A} / \mathrm{B}(\mathrm{a}) \mathrm{A}(\mathrm{r}$ $=0.516)$. The result for correlation analysis between individual PAHs in water from QIRE revealed that the 
compounds were derived from a common anthropogenic origin [27]. The strong positive correlation between $\mathrm{B}(\mathrm{k}) \mathrm{F}$ and chrysene observed in this study may be due to close relatedness in molecular weight [28].

Table 5. Diagnostic ratios of PAHs in water during the dry and wet seasons.

\begin{tabular}{|c|c|c|c|c|c|c|}
\hline & DRYSEASON & & & WET SEASON & & \\
\hline Station & $\Sigma L M W / \Sigma H M$ & $\mathbf{F L} / \mathbf{F L}+\mathbf{P Y}$ & $\mathbf{A N} / \mathbf{A N}+\mathbf{P H}$ & 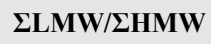 & $\mathbf{F L} / \mathbf{F L}+\mathbf{P Y}$ & $\mathbf{A N} / \mathbf{A N}+\mathbf{P H}$ \\
\hline Okoroutip & 0.0049 & 0.9365 & 0.8960 & 0.0001 & 0.7371 & 0.6484 \\
\hline Ukpenekang & 0.0480 & 0.9951 & 0.4665 & 0.0011 & 0.9545 & 0.6896 \\
\hline Iwochaang & 0.0106 & 0.0125 & 0.1268 & 0.0133 & 0.3007 & 0.4449 \\
\hline Douglas creek & 0.0078 & 0.6923 & 0.1295 & 0.0036 & 0.8279 & 0.0616 \\
\hline Stubb creek & 0.0195 & 0.5300 & 0.1459 & 0.0041 & 0.9654 & 0.1656 \\
\hline EkpeneUkpa & 0.2863 & 0.6370 & 0.2880 & 0.0381 & 0.7110 & 0.2064 \\
\hline
\end{tabular}

$\mathrm{Fl} / \mathrm{FL}+\mathrm{PY}=$ Fluoranthene/Fluoranthene + Pyrene, $\mathrm{AN} / \mathrm{AN}+\mathrm{PH}=$ Anthracene/Anthracene + Phenanthrene

Table 6. Correlation matrix for high molecular weight PAHs in water from QIRE during the dry season.

\begin{tabular}{|c|c|c|c|c|c|c|c|}
\hline & $\mathbf{B}(\mathbf{a}) \mathbf{A}$ & Chrysene & $\mathbf{B}(\mathbf{b}) \mathbf{F}$ & $\mathbf{B}(\mathbf{k}) \mathbf{F}$ & $\mathbf{B}(\mathbf{a}) \mathbf{P}$ & $\operatorname{Ind}(1,2,3-c d) P$ & D(ah)A \\
\hline $\mathrm{B}(\mathrm{a}) \mathrm{A}$ & 1 & & & & & & \\
\hline Chrysene & -0.163 & 1 & & & & & \\
\hline $\mathrm{B}(\mathrm{b}) \mathrm{F}$ & -0.315 & 0.575 & 1 & & & & \\
\hline $\mathrm{B}(\mathrm{k}) \mathrm{F}$ & -0.035 & $0.837^{*}$ & 0.325 & 1 & & & \\
\hline $\mathrm{B}(\mathrm{a}) \mathrm{P}$ & 0.640 & -0.012 & 0.241 & -0.119 & 1 & & \\
\hline $\operatorname{Ind}(1,2,3-\mathrm{cd}) \mathrm{P}$ & -0.268 & $0.942 * *$ & 0.426 & $0.938 * *$ & -0.216 & 1 & \\
\hline $\mathrm{D}(\mathrm{ah}) \mathrm{A}$ & $0.994 * *$ & -0.272 & -0.382 & -0.130 & 0.621 & -0.367 & 1 \\
\hline
\end{tabular}

** Correlation is significant at 0.01 level (2-tailed), * correlation is significant at 0.05 level ( 2 -tailed)

Cluster analysis was used to assess similarities or dissimilarities among sampling sites based on the level of PAHs in water for both the dry and wet season and the results presented in Figures 2 and 3 revealed three principal clusters for both seasons. In the dry season, group one was made up of Stubb creek and Okoroutip with the highest concentration of total PAHs and HMW-PAHs, while the second group was made up of Douglas creek and Iwochang with intermediate concentration and the third group was Ukpenekang and EkpeneUkpa with the lowest concentration. These sites especially the control site was free from industrial activity and the level of PAHs here is low and may be due to domestic waste incineration and sewage only. During the wet season, three clusters were identified belonging to a group of three similar PAHs. The highest total PAHs was recorded by group one (Douglas, Ukpenekang and Stubb creek), while Iwochang and Okoroutip belonged to group two and the control site with the lowest total PAH concentration was classified as group three.

Table 7. Correlation matrix for high molecular weight PAHs in water from QIRE during the wet season.

\begin{tabular}{|c|c|c|c|c|c|c|c|}
\hline & B(a)A & Chrysene & $\mathbf{B}(\mathbf{b}) \mathbf{F}$ & $\mathbf{B}(\mathbf{k}) \mathbf{F}$ & $\mathbf{B}(\mathbf{a}) \mathbf{P}$ & $\operatorname{Ind}(1,2,3-c d) P$ & D(ah)A \\
\hline $\mathrm{B}(\mathrm{a}) \mathrm{A}$ & 1 & & & & & & \\
\hline Chrysene & -0.037 & 1 & & & & & \\
\hline $\mathrm{B}(\mathrm{b}) \mathrm{F}$ & -0.164 & $0.786^{*}$ & 1 & & & & \\
\hline $\mathrm{B}(\mathrm{k}) \mathrm{F}$ & -0.189 & -0.031 & 0.271 & 1 & & & \\
\hline $\mathrm{B}(\mathrm{a}) \mathrm{P}$ & -0.158 & -0.454 & -0.110 & -0.213 & 1 & & \\
\hline $\operatorname{Ind}(1,2,3-\mathrm{cd}) \mathrm{P}$ & 0.188 & -0.411 & 0.033 & 0.368 & $0.719 * *$ & 1 & \\
\hline $\mathrm{D}(\mathrm{ah}) \mathrm{A}$ & $0.516^{*}$ & -0.111 & -0.111 & -0.461 & $0.657^{*}$ & 0.572 & 1 \\
\hline
\end{tabular}

** correlation is significant at 0.01 level ( 2 -tailed), * correlation is significant at 0.05 level ( 2 -tailed)

Hierarchical dendrogram was used to assess similarities between individual PAHs in water from QIRE for the dry and wet seasons are presented in Figures 4 and5. Three primary clusters were identified in both seasons. The first group and second group comprised of $\mathrm{B}(\mathrm{b}) \mathrm{F}$ and Ind (1,2,3-cd)P respectively while the third group comprised of the remaining carcinogenic PAHs. The observed difference in the cluster for the two seasons may be due to the effect of flooding and possible dilution. Water volume and flow rate of the river is usually lower in the dry season which suggests 
higher organic pollution [29]. However, the effect of dilution expected to result in total PAH decrease is compensated for by wet deposition of soot as a result of gas flaring. The grouping in Figures 3 and 4 may be due to the effect of concentration and similar anthropogenic source. Strong positive correlation between PAHs in the third group corroborates with the correlation matrix for water during the wet season. Several authors have reported the high levels of $\mathrm{B}(\mathrm{b}) \mathrm{F}$ and Ind (1,2,3-cd) $\mathrm{P}$ in environmental samples from the Niger delta, which may be anthropogenic $[17,30]$.

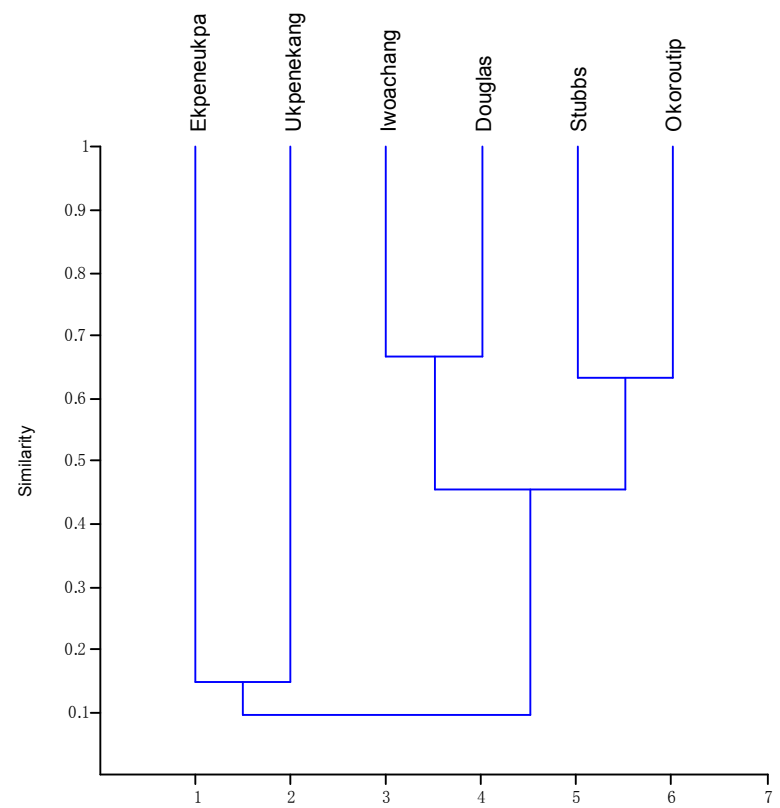

Figure 2. Cluster analysis showing the spatial distribution of PAHs among different sampling sites in water from QIRE during the dry season.

\section{Conclusion}

The level of PAHs in water from QIRE was evaluated in this study. Data analysis using diagnostic ratio, correlation studies and hierarchical cluster analysis was used to identify the sources of PAHs in this study. The total PAHs and total C-PAHs at all the sites except the control site were higher than the standard of European Union and WHO respectively. The values of $\mathrm{B}(\mathrm{b}) \mathrm{F}$ and $\operatorname{Ind}(1,2,3-\mathrm{cd}) \mathrm{P}$ at all the sampling sites during both seasons, were above the WHO standard of 5 $\mathrm{x} 10^{-5} \mathrm{mg} / \mathrm{l}$ for individual PAHs in coastal and surface water. The PAHs detected were predominantly HMW-PAHs predominantly from pyrogenic sources such as gas flaring from and vehicular emissions. Atmospheric deposition may have been the pathway for entry of PAHs into the water bodies. Three primary PAH clusters were identified in both seasons indicating different levels of anthropogenic activities in the cluster areas. The result revealed high levels of $\mathrm{B}(\mathrm{b}) \mathrm{F}$ and(1,2,3-cd)P, two carcinogenic PAHs in lower reach of the Qua Iboe River estuary. Carcinogenic risk assessment of $\mathrm{PAH}$ from the water of QIRE and strict legislation on gas flaring in the area is suggested.

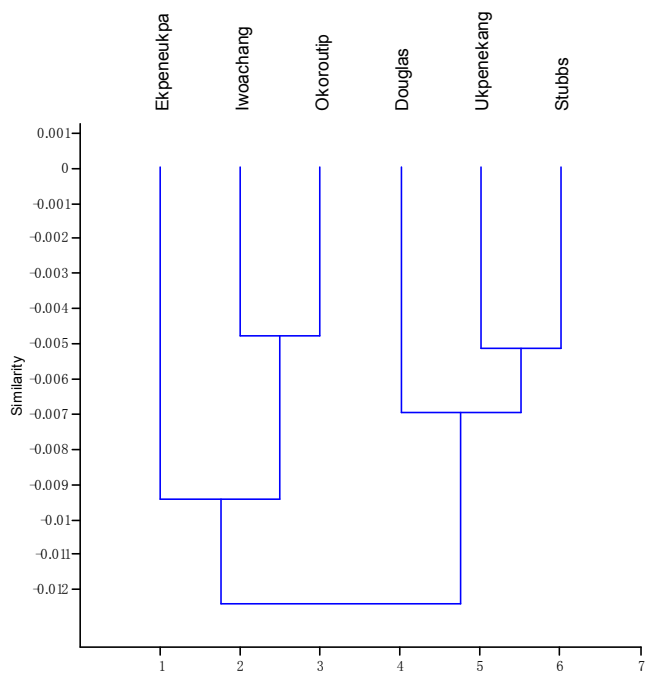

Figure 3. Cluster analysis showing the spatial distribution of PAHs among different sampling sites in water from QIRE during the wet season.

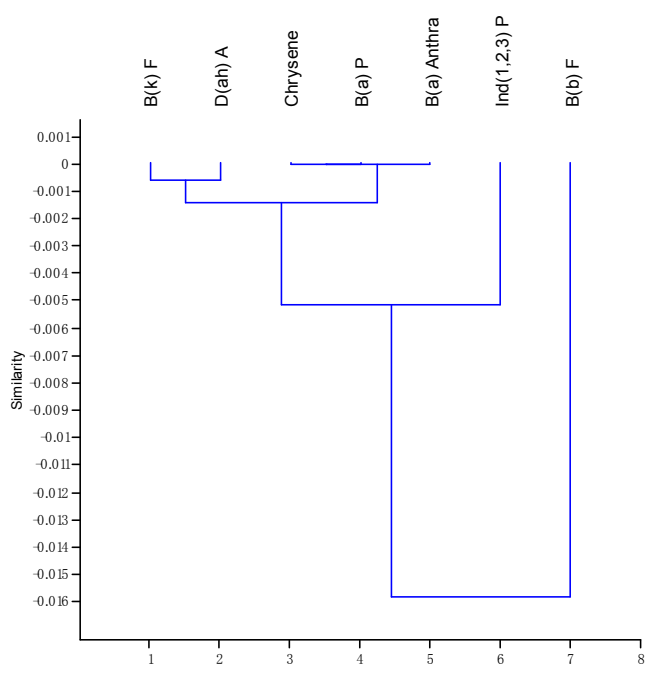

Figure 4. Hierarchical dendrogram for carcinogenic PAHs in water from QIRE during the dry season.

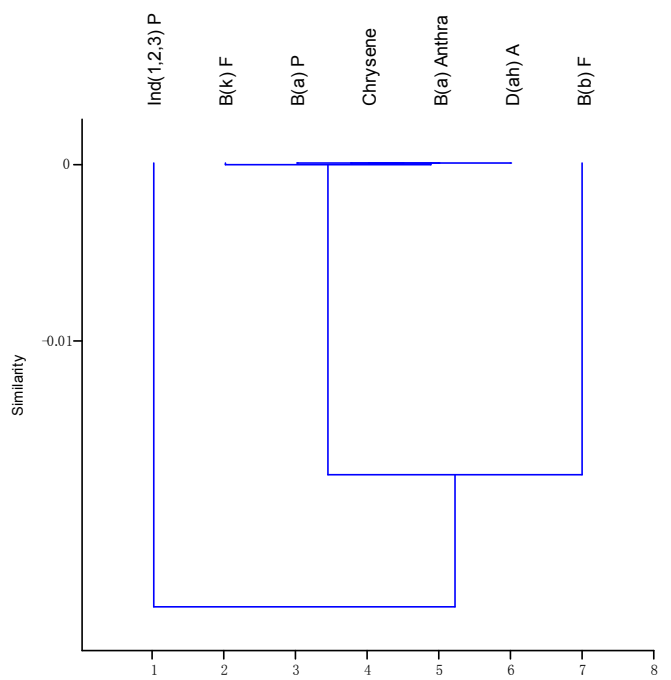

Figure 5. Hierarchical dendrogram for carcinogenic PAHs in water from QIRE during the wet season. 


\section{References}

[1] G. Oze, R. Oze, C. Anunuso, H. Ogukwe, and Okorie, K, "Heavy metal pollution of fish of Qua Iboe river estuary: Possible implication for neurotoxicity," Int. J. Toxicol, vol. 3(1) pp 12-15, 2005.

[2] K. Srogi, "Monitoring of environmental exposure to polycyclic aromatic hydrocarbon: A review. Environ. Chem. Letters," vol. 5, pp 169-195, 2007.

[3] Agency for Toxic Substances and Disease Registry. (ASTDR). "Toxicological profile for polycyclic aromatic hydrocarbons (PAHs)" PB / 95 / 264370. Atlanta, USA. 1995.

[4] G. R. Ana, K. C. Sridher, and O. G. Emerole, "Contamination of surface water by polycyclic aromatic hydrocarbons in two Nigerian coastal communities," J. Environ. Hlth. Res., vol. 11(2) pp 77-86, 2010.

[5] W. Wilcke, "Polycyclic aromatic hydrocarbons (PAHs) in soila review. J. Plant Nut. Soil Sci., vol. 163, pp 229-248, 2000.

[6] Y. Z. Zhang, S. Tao, H. Z. Shen, J. M. Ma, "Inhalation exposure to ambient polycyclic aromatic hydrocarbons and lung cancer risk of Chinese population" Proceedings of the National Academy of Sciences, vol 106, 21063-21067, 2009.

[7] T. O'Neil, Curse of the Black man's gold: Hope and betrayal in the Niger Delta. National Geographic Magazine, p. 12. February, 2007.

[8] A. Sarma, and H. Sarma, "Enhanced biodegradation of oil products by some microbial isolates supplemented with heavy metals," Int. J. Bot., vol. 6: pp 441 - 445, 2011.

[9] R. Nwilo, and O. Badejo, "Oil spill problems and management in the Niger Delta". International oil spill conference, Fluorida, USA, pp 15-19 May, 2005.

[10] Odugwu, "NOSCP and oil spill management," The pioneer, p.10, April 15, 2013.

[11] E. Manoli, and C. Samara, "Polycyclic aromatic hydrocarbon in natural waters: Source, occurrence and analysis," Trends Anal. Chem. vol. 18(6): pp 417 - 420, 1999.

[12] M. A. Gorleku, D. Carboo, L. Palm, W. Quasie, and A. Armah, "Polycyclic aromatic hydrocarbon pollution in marine waters and sediments at Temaharbour, Ghana," Acad. J. of Environ. Sci., vol. 2(7) pp 108-115, 2014.

[13] C. Anyakora, and H. Coker, "Determination of Polycyclic aromatic hydrocarbon in selected water bodies in the Niger Delta,” Afri. J. Biotech., vol. 5(21) pp 2024- 2031, 2006.

[14] D. K. Essumang, C. K. Adokoh, J. Afriyie, and E. Mensah, "Source assessment and analysis of PAHs in the Oblogo waste disposal sites and some water bodies in and around the Accra metropolis of Ghana," J. Water Res. and Prot. vol 1 pp $456-$ 468, 2009.

[15] I. Isine "Akwa Ibom community locks down Exxon Mobil," premium times, July 9, 2014. Assessed on September 172015 at $\mathrm{http} / / \mathrm{www}$. premiumtimesng.com/regional/sso.

[16] E. Caylak, "Health risk assessment for trace metals, polycyclic aromatic hydrocarbons and trihalomethanes in drinking water of Cankiri, Turkey," E-Journal of chemistry, vol 9(4): pp 1916-1991, 2012.
[17] E. Inam, E. Owhoeke, and J. Essien, "Human carcinogenic risk assessment of polycyclic aromatic hydrocarbon in freshwater samples from Ogba/ Egbema / Andoni communities in River state, Nigeria," J. Chem. Soc. Nig. vol 39 (2) pp 15 -22. 2014.

[18] C. Anyakora and H. Coker, "Assessment of Polycyclic aromatic hydrocarbon contamination threat on groundwater: A case study of the Niger Delta region of Nigeria," Int. J. Risk Assess. Mgt., vol 13(2) pp 150- 161, 2009.

[19] J. Li, X. Shang Z. Zhao, R. L. Tanguay, Q. Dong and C. Huang, "Polycyclic aromatic hydrocarbons in water, sediment, soil, and plants of the Aojiang River waterway in Wenzhou, China," J. Hazard Mater. vol 173(1-3) pp75-81, January, 2010.

[20] R. J. Law, V. I. Dawes, R. J. Woodhead, and P. Matthissen, "Polycyclic aromatic hydrocarbons (PAHS) in seawater around England and Wales," Mar. Poll. Bull., vol 34(5) pp 306-322, 1997.

[21] H. H. Soclo, P. Grrigue, and M. Ewald, Origin of Polycyclic aromatic hydrocarbons (PAHs) in coastal marine sediments: Case studies in Cotonou (Benin) and Aquitaine (France) areas," Mar. Pollut. Bull., vol 40(5) pp 387-396, 2000.

[22] M. B. Yunker, R. W. Macdonald, R. Vingerzan, R H. Mitchell, D. Goyette, and S. Sylvestre, "PAHs in the Fraser River basin: A critical appraisal of PAH ratio as indicators of PAH source and composition," Organic Geochemistry, vol. 33(4) pp 489$515,2002$.

[23] Z. Zhang, J. Huang, G. Yu, and H. Hong, "Occurrence of PAHs, PCBs and organochloride pesticides in the Tonghui River of Beijing, China," Environmental Pollution, vol. 130(2) pp 249-261, 2004.

[24] M. Tobiszewski, and J. Namiesnik," PAH diagnostic ratios for the identification of pollution emission sources," Env. Poll. vol. 162 pp 110-119, 2012.

[25] K. Opuene, I. E. Agbozu, O. Adegboro, “A critical appraisal of PAHs indices as indicators of PAHs source and composition of Elelenwo creek, southern Nigeria," Environmentalist, vol. 29 pp 47-55, 2009.

[26] M. Sinae, and A. Mashinchian, "Polycyclic aromatic hydrocarbons in the coastal sea water, the surface sediment and mudskipper (Boleophthalmusdussumer) from coastal areas of Persian Gulf: Source investigation, composition pattern and spatial distribution," J. Environ. Health Sci. Engr. vol. 12 pp $59-63,2014$.

[27] E. Gilbert, D. Dadoo, R. Okai-Sam, D. Essumang, and K. Quagraine, "Characterization and source assessment of heavy metals and PAHs in sediment of Fosu Lagoon, Ghana," J. Environ. Sci. Hlth. vol. 41 pp 2447- 2755, 2006.

[28] D. H. Ogbuagu, C. G. Okoli, C. L. Gilbert, and S. L. Madu, "Determination of the concentration of groundwater sources in Okrika mainland with polycyclic aromatic hydrocarbons," British J. Environ. Clim. Change, vol. 4(3) pp 90-102, 2011.

[29] S. Farooq, S. Equani, R. Malik, Y. Zhang, J. Li, L. Xang, K. Jones, and Z. Shinwari, "Occurrence, fingerprinting and ecological risk assessment of PAHs in Chenab river," Parkistan. J. Environ. Monit. vol. 13 pp 3207- 3215, 2011. 
[30] K. W. Nkpa, M. O. Wegwu, and E. B. Essien, "Assessment of polycyclic hydrocarbons (PAHs) levels in two commercially important fish species from crude oil polluted waters of Ogoni-land and their carcinogenic health risks," J. Env. And Earth Sci. vol. 3(8) pp 128- 137, 2013. 